\title{
Dual Active Galactic Nuclei
}

\section{Jenny E. Greene ${ }^{1}$, Fransisco Mueller-Sanchez ${ }^{2}$ and Julia M. Comerford ${ }^{2}$}

\author{
${ }^{1}$ Department of Astrophysics, Princeton University, Princeton, NJ 08544, USA \\ ${ }^{2}$ Department of Astrophysical and Planetary Sciences, University of Colorado, Boulder, \\ CO 80309, USA
}

\begin{abstract}
We still do not know the timescale for the merging of binary black holes (BHs). This timescale has important implications for gravitational wave predictions and our understanding of $\mathrm{BH}$ demographics. Here we discuss efforts to constrain the fraction of BH pairs on kpc scales using observations of dual active galactic nuclei.
\end{abstract}

Keywords. supermassive black holes, active galactic nuclei, binary black holes

\section{Introduction}

We live in a hierarchical universe, where structures collapse as small units and then grow by merging. We see direct evidence of these mergers in the galaxy population. We also know that most, if not all, massive galaxies today harbor supermassive black holes (BHs) at their centers. The question then becomes, what happens to the pair of BHs during and after a merger?

We know that the two BHs will sink to the center of the potential via dynamical friction, and then the binary will harden via three-body interactions with stars. However, in an axisymmetric potential, at separations of $\sim 1 \mathrm{pc}$ it is possible that the BHs may run out of stars to scatter, and stall (e.g., Begelman et al. 1980; Quinlan 1996). In principle, there may be pc-scale binary BHs lurking in the centers of many galaxies. From a theoretical perspective, thirty years of debate has not settled the issue of whether binary BHs stall (e.g., Gould \& Rix 2000; Merritt \& Poon 2004; Yu et al. 2005; Armitage \& Natarajan 2005; Merritt \& Milosavljević 2005; Tremmel et al. 2015). Observationally we cannot rule out that BHs stall at pc scales, because it is so difficult to spatially resolve pairs of BHs on such small scales. There is really only one iron-clad case of a binary BH with a $7 \mathrm{pc}$ separation where we have imaging of a BH pair (Maness et al. 2004; Rodriguez et al. 2006).

However, many things depend on the timescale for binary BH mergers. For one thing, the detectability of low-frequency gravitational radiation (e.g., with pulsar timing arrays; see other contributions in this volume) depends sensitively on the merger timescale of $\mathrm{BH}$ binaries. The overall growth rate of $\mathrm{BHs}$ and the distribution of $\mathrm{BH}$ spin depends on the merger rate (e.g., Volonteri et al. 2005). The importance of the so-called gravitational slingshot effect, in which anisotropic gravitational radiation from unequal $\mathrm{BH}$ binaries imparts a net linear momentum to a merged remnant, also depends on the merger timescale. While people have looked for ejected BHs (e.g., Hoffman \& Loeb 2006; Civano et al. 2010), it is not clear that any have been found.

This article takes a step back from pc-scale binary BHs and asks whether we can put interesting constraints on the $\mathrm{BH}$ merger rate using observations of pairs of $\mathrm{BHs}$ on $\mathrm{kpc}$ scales. These so-called dual AGN are special cases where a pair of BHs are both activated presumably during or following a merger. A number of studies have shown that a galaxy is more likely to be an AGN if it is in a galaxy pair with separations of 5-20 kpc (Ellison 

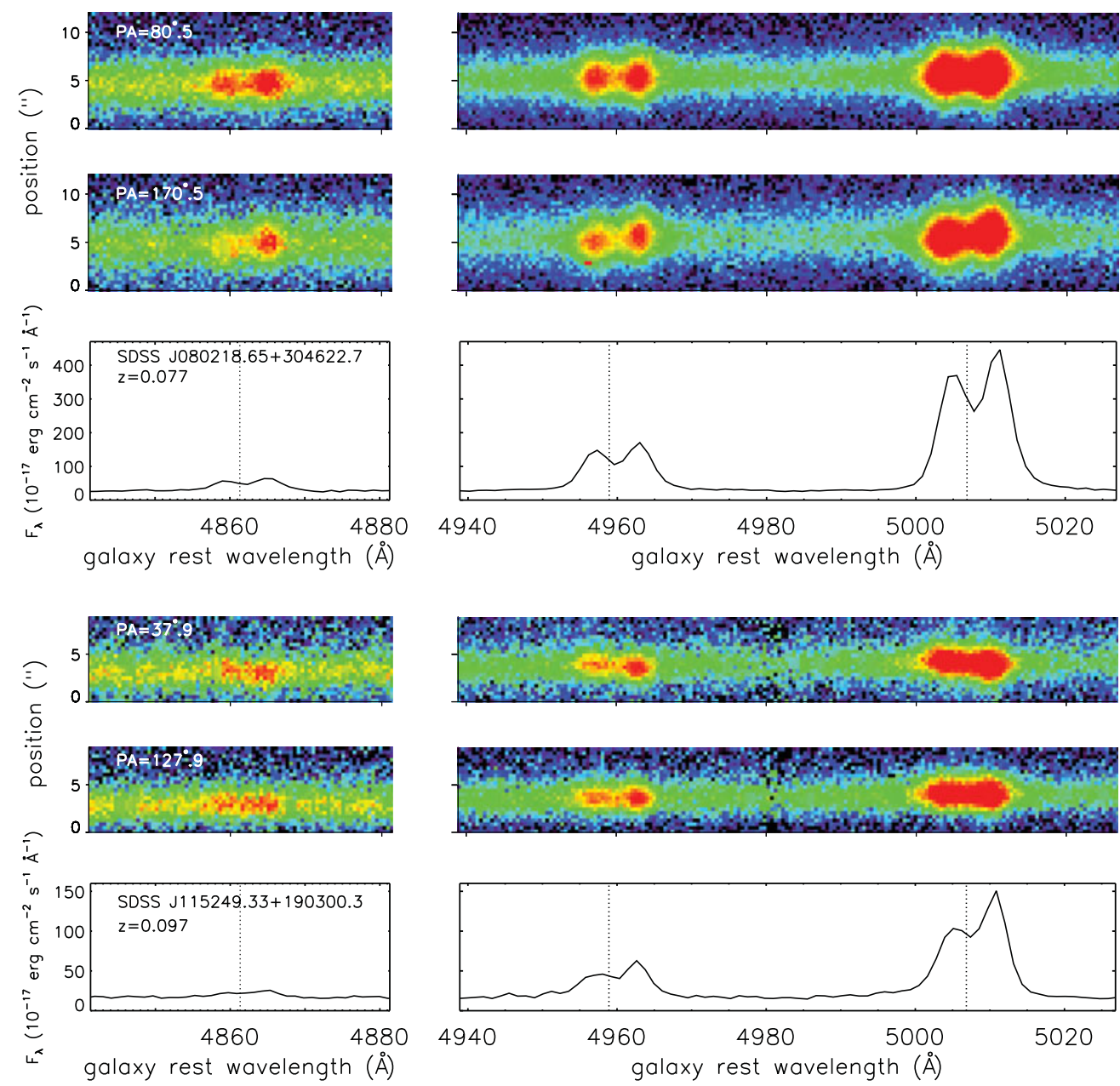

Figure 1. Example two-dimensional spectra (color) and one-dimensional SDSS spectra for some probably dual AGN adapted from (Comerford et al. 2009a).

et al. 2011; Silverman et al. 2011). However, to probe yet smaller scales (<kpc) is difficult with ground-based imaging, corresponding to sub-arcsecond separations at $z=0.1$.

We will briefly summarize observational programs to find dual AGN with $\sim \mathrm{kpc}$ separations. We will then focus on the close cousins of dual AGN, the offset AGN. Then we will discuss future searches. Finally, we discuss one spectroscopic method for putting limits on binary BHs.

\section{Search for Dual Active Galaxies}

Pairs of accreting BHs with kpc separations could be distinguished in a number of ways. For instance, one might find two blue nuclei, pointing to a pair of unobscured quasars (e.g., Myers et al. 2008; Hennawi et al. 2010; Liu et al. 2011). Alternately, one might find two X-ray or radio cores (e.g., Rodriguez et al. 2006). Finally, one might identify them spectroscopically, via their orbital motions. A handful of such objects were known (e.g., Komossa et al. 2003; Koss et al. 2011). The Sloan Digital Sky Survey (SDSS, York et al. 2000) performed a uniform survey of galaxies and AGN, which allows for spectroscopic 

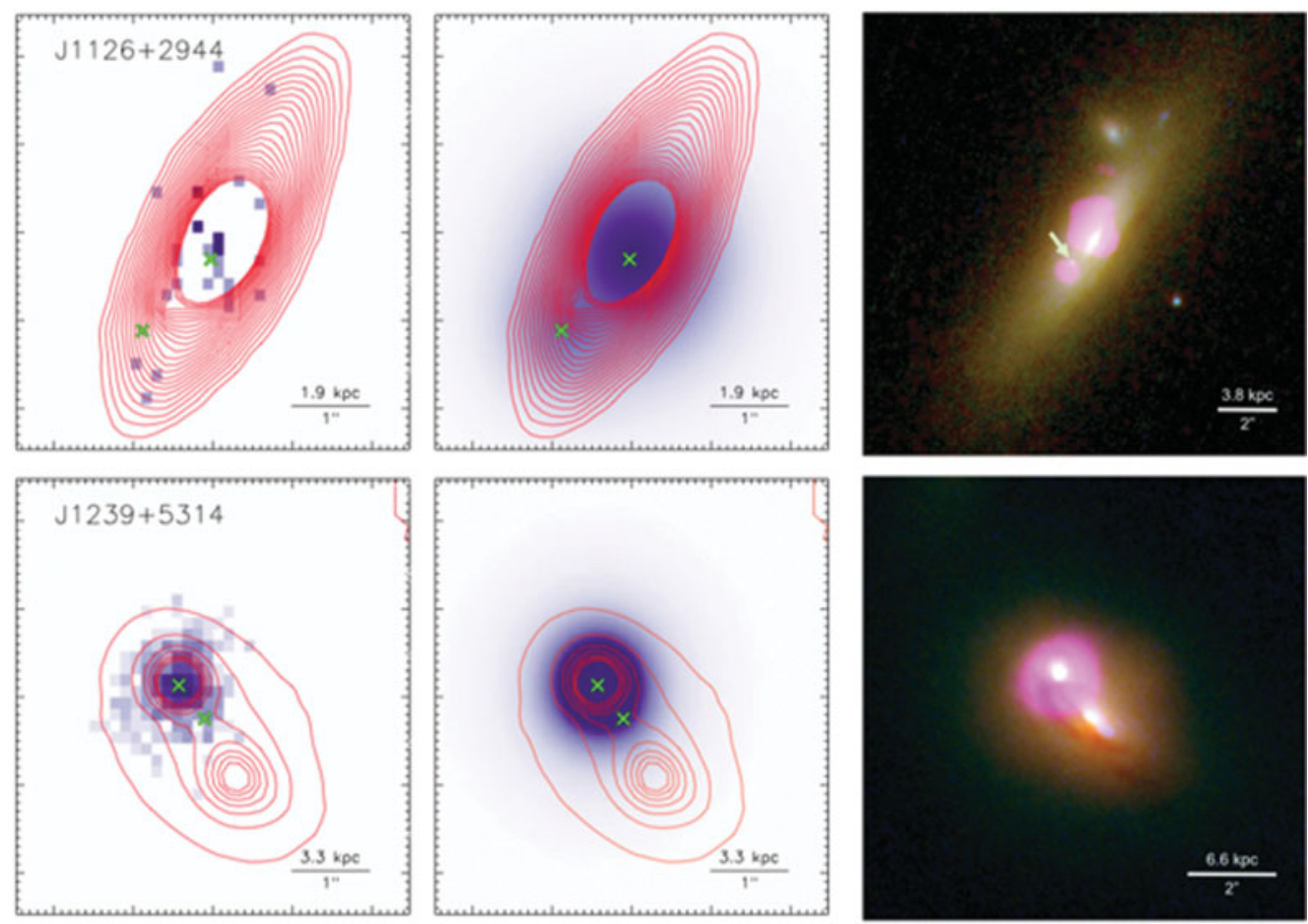

Figure 2. Dual AGN system (top) and an offset or dual AGN system (bottom) identified from follow-up Chandra observations, adapted from Comerford et al. (2015).

selection of possible dual AGN. Specifically, orbital motion could lead to velocity splitting of the narrow emission lines for kpc-scale separations (Figure 1).

\subsection{Spectroscopic Samples}

In fact, $\sim 10 \%$ of low-redshift AGN show velocity splitting in their narrow emission lines (Heckman et al. 1981; Veilleux 1991; Comerford et al. 2009a; Wang et al. 2009; Liu et al. 2010b; Smith et al. 2010). Examples of these "double-peaked" AGN are shown in Figure 1. Hundreds of potential dual AGN were found in SDSS. The next obvious question becomes, are these in fact AGN pairs, or are the multiple velocity peaks in the SDSS spectra caused by other things. Recall that the SDSS I/II fibers were $3^{\prime \prime}$ in diameter, encompassing roughly $5 \mathrm{kpc}$ at the typical redshift of $z \approx 0.1$ of the SDSS main galaxy sample. Thus, outflows and rotating disks make significant contributions to the double peaked sample (Crenshaw et al. 2010).

The first attempts to determine the nature of these sources used long-slit spectroscopy and NIR imaging (Liu et al. 2010a; Shen et al. 2011; McGurk et al. 2011; Comerford et al. 2012; Ge et al. 2012). Roughly 10\% of double-peaked systems have line emission aligned with two NIR continuum peaks that indicate bona-fide dual systems. This translates to $1 \%$ of all low-redshift AGN in SDSS being part of a dual AGN system.

\subsection{X-ray Follow-up}

In principle, hard X-rays should be able to penetrate even very significant absorption and provide a more reliable view of dual AGN (Figure 2). In practice, the X-ray luminosities have proved systematically lower than expected, making X-ray followup far more expensive than expected (Comerford et al. 2011; Liu et al. 2013; Comerford et al. 2015). 

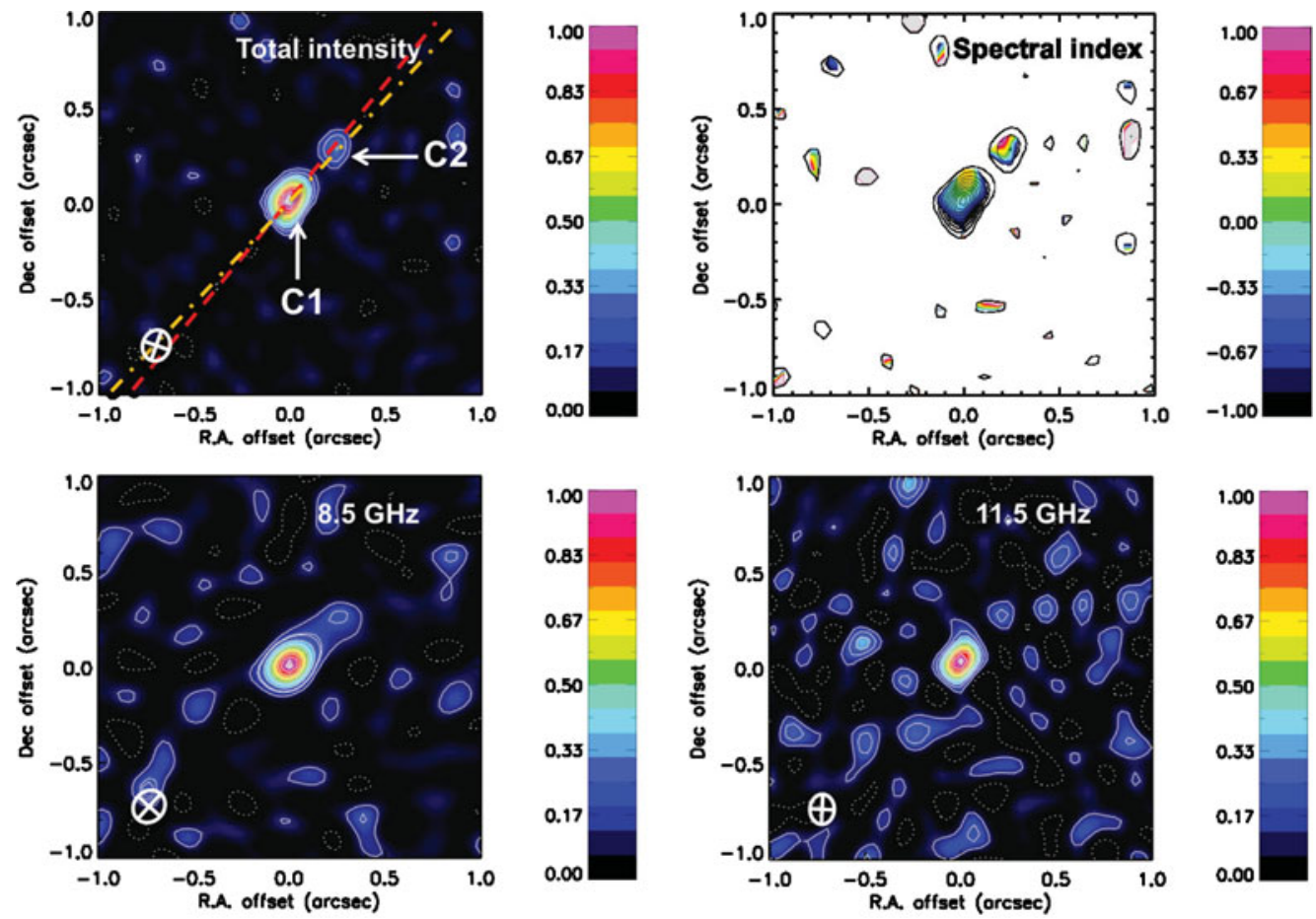

Figure 3. Compelling dual AGN system based on follow-up JVLA observations, adapted from Muller-Sanchez et al. (2015).

\subsection{Radio Follow-up}

With the advent of the EVLA, radio follow-up became a very efficient method to search for true dual AGN (Rosario et al. 2010; Fu et al. 2011; An et al. 2013). Müller-Sanchez et al. (2015) have just performed a uniform survey of double-peaked AGN using EVLA, to find a dual AGN rate of $\sim 10-15 \%$ (Figure 3). This rate is roughly consistent with the earlier spectroscopic follow-up studies, but is likely more robust, since from the radio imaging + spectroscopy it is possible to find likely jets as well as dual cores.

\section{Offset AGN}

A complementary approach to understanding the role of merging in AGN triggering and $\mathrm{BH}$ growth is to look for cases of velocity offsets between stellar absorption features and AGN emission lines (see examples in Figure 4). These offset AGN may be a signpost of an inspiraling accreting BH on kpc scales. There have been a few serendipitous detections of such systems (Barth et al. 2008; Bianchi et al. 2013). However, Comerford et al. (2009a) performed the first systematic search for such systems at intermediate redshift using the DEEP2 survey. They found velocity offsets were quite common (30\%) at these redshifts. To see whether this trend persists at lower redshift, she performed two additional searches (Comerford et al. 2013; Comerford \& Greene 2014). Follow-up of these sources is ongoing, but the initial estimate is that the offset AGN fraction increases from $2 \%-30 \%$ from the present to $z \sim 0.7$. 

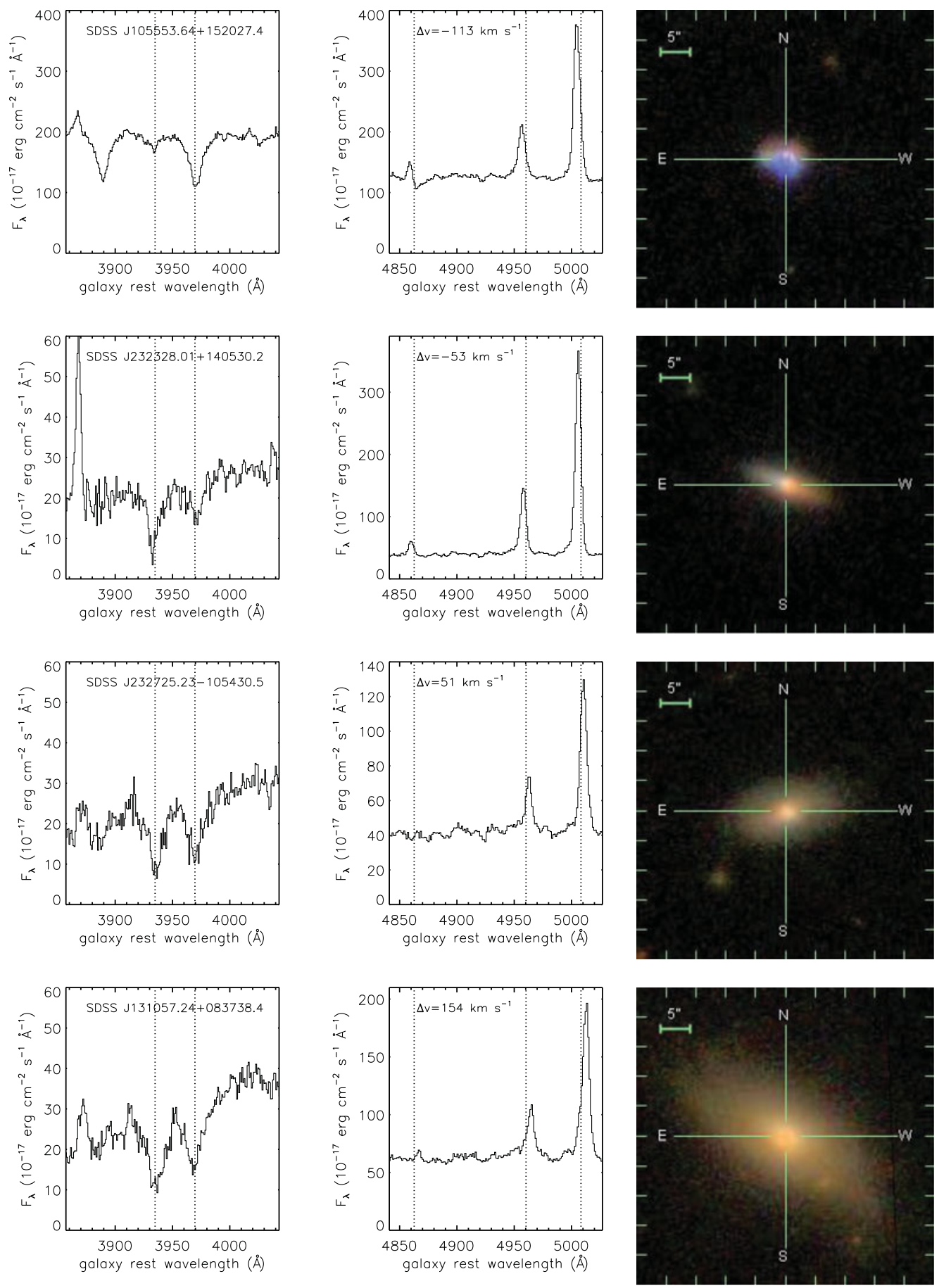

Figure 4. Examples of offset AGN candidates, showing that the narrow emission lines show consistent velocity offsets when compared with the stellar absorption lines. Adapted from Comerford \& Greene (2014). 


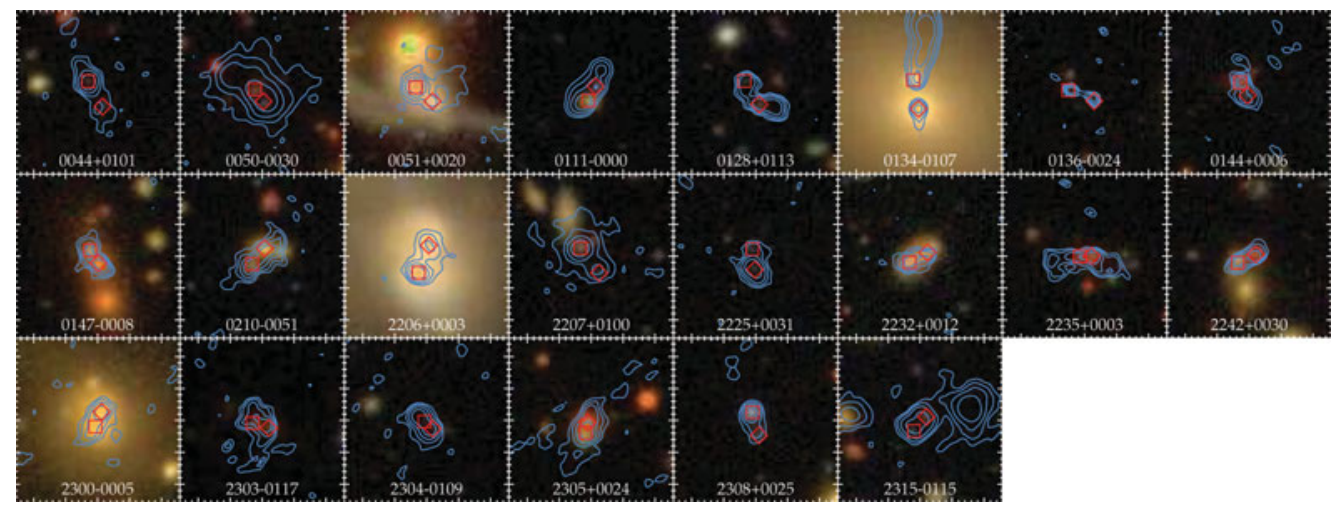

Figure 5. The possibility of finding dual AGN in the radio directly adapted from Fu et al. (2015). From a pilot follow-up survey, Fu et al. estimate $60 \%$ of their candidates will be true dual AGN.

\section{The Future}

While double-peaked sources are intriguing, they have not proved a very efficient method for finding dual AGN (only 10\% are true dual AGN). It is interesting to think about alternate search methods. One is to start by detecting dual galaxy cores, and then following these up to see how many harbor dual AGN (Comerford et al. 2009b, 2011; Lackner et al. 2014). Another is to search directly in the radio for dual cores (Fu et al. 2015, Figure 5). It is early days for these alternate searches, so stay tuned for the true dual AGN space density!

\section{References}

An, T., Paragi, Z., Frey, S., et al. 2013, MNRAS, 433, 1161

Armitage, P. J. \& Natarajan, P. 2005, ApJ, 634, 921

Barth, A. J., Greene, J. E., \& Ho, L. C. 2008, AJ, 136, 1179

Begelman, M. C., Blandford, R. D., \& Rees, M. J. 1980, Nature, 287, 307

Bianchi, S., Piconcelli, E., Pérez-Torres, M. Á., et al. 2013, MNRAS, 435, 2335

Civano, F., et al. 2010, ApJ, 717, 209

Comerford, J. M., Gerke, B. F., Newman, J. A., et al. 2009a, ApJ, 698, 956

Comerford, J. M., Griffith, R. L., Gerke, B. F., et al. 2009b, ApJ, 702, L82

Comerford, J. M., Gerke, B. F., Stern, D., et al. 2012, ApJ, 753, 42

Comerford, J. M. \& Greene, J. E. 2014, ApJ, 789, 112

Comerford, J. M., Pooley, D., Barrows, R. S., et al. 2015, ApJ, 806, 219

Comerford, J. M., Pooley, D., Gerke, B. F., \& Madejski, G. M. 2011, ApJ, 737, L19

Comerford, J. M., Schluns, K., Greene, J. E., \& Cool, R. J. 2013, ApJ, 777, 64

Crenshaw, D. M., Schmitt, H. R., Kraemer, S. B., Mushotzky, R. F., \& Dunn, J. P. 2010, ApJ, 708,419

Ellison, S. L., Patton, D. R., Mendel, J. T., \& Scudder, J. M. 2011, MNRAS, 418, 2043

Fu, H., Zhang, Z.-Y., Assef, R. J., et al. 2011, ApJ, 740, L44

Fu, H., Myers, A. D., Djorgovski, S. G., et al. 2015, ApJ, 799, 72

Ge, J.-Q., Hu, C., Wang, J.-M., Bai, J.-M., \& Zhang, S. 2012, ApJS, 201, 31

Gould, A. \& Rix, H.-W. 2000, ApJ, 532, L29

Heckman, T. M., Miley, G. K., van Breugel, W. J. M., \& Butcher, H. R. 1981, ApJ, 247, 403

Hoffman, L. \& Loeb, A. 2006, ApJ, 638, L75

Lackner, C. N., et al. 2014, AJ, 148, 137

Liu, X., Greene, J. E., Shen, Y., \& Strauss, M. A. 2010a, ApJ, 715, L30

Liu, X., Shen, Y., Strauss, M. A., \& Greene, J. E. 2010b, ApJ, 708, 427 
Liu, X., Civano, F., Shen, Y., et al. 2013, ApJ, 762, 110

Maness, H. L., Taylor, G. B., Zavala, R. T., Peck, A. B., \& Pollack, L. K. 2004, ApJ, 602, 123

McGurk, R. C., Max, C. E., Rosario, D. J., et al. 2011, ApJ, 738, L2

Merritt, D. \& Milosavljević, M. 2005, Living Rev. Relativ., 8, 8

Merritt, D. \& Poon, M. Y. 2004, ApJ, 606, 788

Müller-Sanchez, F., Comerford, J. M., Nevin, R., et al. 2015, ApJ, in press; arXiv:1509.04291

Quinlan, G. D. 1996, New Astron., 1, 35

Rodriguez, C., Taylor, G. B., Zavala, R. T., et al. 2006, ApJ, 646, 49

Rosario, D. J., Shields, G. A., Taylor, G. B., Salviander, S., \& Smith, K. L. 2010, ApJ, 716, 131

Shen, Y., Liu, X., Greene, J. E., \& Strauss, M. A. 2011, ApJ, 735, 48

Silverman, J. D., et al. 2011, ApJ, 743, 2

Smith, K. L., Shields, G. A., Bonning, E. W., et al. 2010, ApJ, 716, 866

Tremmel, M., Governato, F., Volonteri, M., \& Quinn, T. R. 2015, MNRAS, 451, 1868

Veilleux, S. 1991, ApJ, 369, 331

Volonteri, M., Madau, P., Quataert, E., \& Rees, M. J. 2005, ApJ, 620, 69

Wang, J.-G., Dong, X.-B., Wang, T.-G., et al. 2009, ApJ, 707, 1334

Yu, Q., Lu, Y., \& Kauffmann, G. 2005, ApJ, 634, 901 